\title{
Tecnura
}

\section{Una revisión de plataformas robóticas para el sector de la construcción}

\author{
Robotics platforms for the construction sector: A review
}

\author{
Yeyson Alejandro Becerra Mora ${ }^{1}$ (D)
}

Cómo citar: Becerra Mora, Y.A. (2020). Una revisión de plataformas robóticas para el sector de la construcción Tecnura. Tecnura, 24(63) 115-132. DOI: https://doi.org/10.14483/22487638.15384

Fecha de de recepción: 10 de junio de 2019

\section{RESUMEN}

Contexto: En los últimos años la robótica ha venido integrándose a los distintos sectores económicos, permitiendo optimizar recursos y tiempos de producción; así como reducir los riesgos a los que se puede enfrentar el humano en su entorno de trabajo. Uno de los sectores más dinámicos y complejos para los sistemas autónomos es el de la construcción; razón por la cual se ha hecho pocos adelantos en los últimos años. En este trabajo se presenta una revisión de trabajos realizados para el sector de la construcción mediante sistemas robóticos.

Método: Este trabajo presenta inicialmente las ventajas y desventajas que tienen los robots en entornos de construcción. Posteriormente se divide en tres categorías los diversos trabajos presentados; la primera se enfoca en aquellos sistemas que fabrican piezas o módulos con geometrías complejas en entornos fuera del área de construcción; la segunda muestra sistemas retroalimentados para entornos cambiantes y algoritmos de planificación de rutas utilizados para el posi-
Fecha de de aceptación: 18 de octubre de 2019

cionamiento del efector final; y la última, robots que realizan tareas pesadas o repetitivas para el beneficio del ser humano.

Resultados: Se obtiene un panorama de los diversos trabajos que se han hecho en robótica para el sector de la construcción; conociendo los actuales sistemas robóticos y los elementos que los componen para el desarrollo de tareas en este entorno.

Conclusiones: Los brazos robóticos que fueron inicialmente empleados en el sector de la industria llegan al sector de la construcción para desarrollar superficies complejas que involucren la optimización de recursos y tiempo. Este sector ha empezado a atraer recurso humano con habilidades en robótica e inteligencia artificial, haciendo que estas labores sean interdisciplinares. Las proyecciones muestran que el ser humano reducirá los riesgos de lesiones en este sector, ya no tendrá que realizar tareas repetitivas o que involucren fuerza, debido a que el robot se encargará de ellas. Palabras clave: robótica; construcción; arquitectura; geometrías complejas.

1 Ingeniero en Automatización, máster en Automática y Robótica. Docente de la Corporación Unificada Nacional de Educación Superior (CUN). Bogotá, Colombia. Contacto: yeyson_becerra@cun.edu.co

ORCID: https://orcid.org/0000-0002-6718-4962 


\section{ABSTRACT}

Context: In recent years, robotics has been integrated into different economic sectors, allowing resources and production time to be optimized; as well as reducing the risks that humans can face in their work space. One of the most dynamic and complex sectors for the autonomous systems, is construction sector; which is why little progress has been made in the last years. This paper presents a review of studies for the construction sector using robotic systems.

Method: This review initially presents the advantages and disadvantages that robots have in construction environments. Subsequently, the different studies presented are divided into three categories; the first focuses on those systems that allow for off-site manufacturing, like building modules and pieces with complex geometry; the second shows feedback systems for dynamic environments and path planning algorithms used for positioning the end-effector; and the last one, robots that perform heavy or repetitive tasks for the benefit of the human being.
Results: An overview of the different studies that have been done in robotics for the construction sector is obtained; knowing state of the art robotic systems and the elements that compose them for the development of tasks in this environment.

Conclusions: The robotic arms were initially designed to be used in the industry sector, nevertheless, recently they are being used in the construction sector to develop complex surfaces that involve the optimization of resources and time. Furthermore, this sector has begun to attract human resources with skills in robotics and artificial intelligence, making construction tasks, interdisciplinary. The projections show that the human beings will reduce the risks of injuries in this sector; they will no longer have to perform repetitive or heavy tasks, because the robot will take care of them.

Keywords: robotics; construction; architecture; complex geometries.

\section{INTRODUCCIÓN}

Hasta hace algunos años, la industria de la construcción presentaba una gran cantidad de procesos manuales, comprometiendo la seguridad y bienestar del trabajador en muchos de ellos. Estadísticas de los últimos años muestran que existe una gran demanda para cubrir vacantes en labores de construcción, impidiendo que este sector crezca a su potencial. Estas dos principales razones han permitido el ingreso de la robótica al sector de la construcción; con el propósito de ayudar a remplazar o mejorar procesos existentes, y hacerlos más precisos, eficientes y óptimos.

Como se mencionó previamente, diversos países alrededor del mundo están viendo problemas en contratar mano de obra calificada; debido a que se está presentando una menor oferta para cubrir vacantes en este sector. Además, en la actualidad las nuevas generaciones no se están interesando en trabajos donde se utilice más la fuerza que el cerebro. Por esta razón, la robótica se presenta como una solución ante ello, para que este sector continúe creciendo y construya más con menos recursos.

Un ejemplo de lo previamente escrito se presenta en Japón, país que ha disminuido el número de trabajadores en el sector de la construcción. Estadísticas muestran que la edad promedio de ellos se encuentra en los 54 años. Esto claramente muestra una problemática ya que muchos de ellos se jubilarán próximamente, creando una deficiencia de mano de obra en este sector. Lo más preocupante es que los trabajadores que están próximos a retirarse no van a ser reemplazados por jóvenes constructores, ya que los menores de 30 años solo ocupan en torno de un $10 \%$ de 
la fuerza laboral en el sector. Otro ejemplo es Estados Unidos, el cual también está presentando el mismo fenómeno que Japón; la fuerza laboral en el sector de la construcción se ha venido envejeciendo en la última década, según lo afirma la compañía Advanced Construction Robotics. En la actualidad hay problemas para encontrar personal calificado para este tipo de labores y más aún con el desinterés que muestran las generaciones más jóvenes para estos cargos.

Se debe tener en cuenta que el sector de la construcción es de los menos automatizados actualmente, pues tiene como fuente de productividad la mano de obra humana; por lo cual se entiende que las tareas de construcción son complicadas para ser automatizadas. Un ejemplo de ello para entender lo mencionado es que un robot industrial opera en entornos controlados, mientras que un robot en el sector de la construcción debe enfrentarse a entornos cambiantes. Lo cual lleva a que estos últimos deban adaptarse a cambios constantes en su sitio de trabajo, evitando la reprogramación de ellos en la medida de lo posible, con el fin de llegar a ser productivos y rentables.

Actualmente se están empleando brazos robóticos industriales que permiten colaborar en diversas tareas de la construcción; sin embargo, se debe tener en cuenta que diferencias entre la superficie de un laboratorio (entorno controlado) y la del sitio de la construcción pueden ser considerables (entorno cambiante). La intervención del humano en tareas colaborativas con un robot industrial es crucial, ya que en ocasiones este último debe ser intervenido por posibles fallas que presente en la construcción; sin embargo, la precisión que alcanza un robot es casi imposible de ser replicarda por el humano.

Los sitios de construcción tienden a ser desordenados y en constante cambio, razón por la cual se vuelve un desafío operar robots en estos entornos. Un estudio realizado por $\mathrm{ABI}$ Research indica que en 2018 se encontró que el $28,7 \%$ de los robots comerciales tenían algún tipo navegación autónoma, la cual se ha vuelto relevante para la robótica en la construcción; así mismo, este también predice que para 2027, el porcentaje aumentará hasta el 79,3\% (ABI Research, 2019).

El presente artículo se divide en cinco secciones: la primera muestra una serie de ventajas y desafíos que se han identificado para los robots en el sector de la construcción; la segunda presenta diversas plataformas robóticas que se encargan de fabricar geometrías de difícil construcción para el ser humano; la tercera plantea la relevancia de los sensores para los sistemas robóticos y cómo estos colaboran a la toma de decisiones en un entorno cambiante; la cuarta muestra robots robustos para tareas de demolición, levantamiento de cargas pesadas u oficios riesgosos; la última sección sugiere algunas conclusiones.

\section{VENTAJAS Y DESAFÍOS DE LA ROBÓTICA EN EL SECTOR CONSTRUCCIÓN}

Adoptar robots en el sector de la construcción puede traer diversas ventajas, una de ellas es bajar costos de operación a través de recortes en la carga de trabajo necesaria para la obra. Albañiles y obreros que trabajen junto a robots colaborativos pueden incrementar la productividad en la construcción; haciendo que estos últimos realicen las tareas tediosas, repetitivas y pesadas. A diferencia de lo que se podría pensar, los robots llegan a ser ayudantes de los humanos y no a tomar la totalidad de los puestos de trabajo en este sector. Por tanto, un cambio en las relaciones y colaboraciones presentadas en el lugar de trabajo está emergiendo en la actualidad.

Los robots también pueden incrementar la eficiencia en tareas repetitivas; mientras un humano tiene que lidiar con diversos elementos, como agotamiento, aburrimiento, 
problemas personales, errores, pereza, entre otros; un robot, no. Esto hace que la calidad de trabajo del humano se vea comprometida. Un robot puede operar por periodos mucho más prolongados que los de un humano (24 horas/día - 7 días/semana), sin verse comprometida la calidad de sus labores; solo teniendo en cuenta mantenimiento o posibles fallas que se puedan presentar en el sistema. Además, los robots pueden llegar a ser muy precisos en diversas tareas (ensamblaje de piezas, soldado, fresado, manipulación, perforación, entre otras).

Con esta tecnología es posible crear proyectos personalizados y que respondan a la demanda del mercado. Esto permite prefabricar componentes en el lugar de la construcción, evitando la necesidad de transportar materiales o bienes de dimensiones considerables. Por tanto, tiempo y costos del proyecto se reducen; además, cambios imprevistos que pueda sugerir el cliente se pueden realizar rápidamente y con una mínima pérdida de recursos.

Mejorar la movilidad, incrementar la fuerza $\mathrm{o}$, incluso, reducir accidentes asociados al trabajo pueden ser posibles gracias al uso de exoesqueletos. Esta es otra ventaja que ofrece la robótica en el sector de la construcción, haciendo que el trabajador no se vea sometido a altos niveles de estrés causados por labores físicas exigentes.

La robótica reduce el riesgo de accidentes entre los trabajadores de obra, ya que las tareas que comprometan la seguridad del humano pueden ser asignadas a los robots. Eso hace que el número de demandas que pueden ser interpuestas por los mismos trabajadores a la compañía de construcción debido a accidentes laborales, se vean reducidas considerablemente. Además, los robots tienen la ventaja de trabajar en entornos riesgosos (alturas, espacios reducidos, oscuridad, aire contaminado, etc.).
La logística y gestión de una obra también puede ser mejorada a través de robots móviles, los cuales, a su vez, puedan comunicarse con otros dispositivos para solicitar información. Esto permitirá mejorar en tareas colaborativas y seguridad en el equipo de trabajo. Por tanto, mejorar la conectividad a través de redes $5 \mathrm{G}$, hará que los robots mejoren sus habilidades de comunicación y cooperación entre ellos. Para desarrollar una tarea específica en una obra, diversos robots pueden trabajar juntos en distintas subtareas, para completar el trabajo en un menor tiempo. Además, trabajar en enjambre permitirá que todos los robots aprendan de lo que uno de ellos está aprendiendo, haciéndolos más listos y asequibles.

Por otro lado, aún se presentan algunas desventajas en la actualidad para el uso masivo de la robótica en el sector de la construcción. Los brazos robóticos fueron originalmente pensados para ser operados en entornos controlados y así orientados al sector industrial. En el sector de la construcción, cada espacio es único, además de desorganizado por los diversos elementos que se pueden encontrar alrededor. Lo cual exige una mayor autonomía en estos sistemas, haciendo de los sensores, sistemas de control y la inteligencia artificial, elementos claves para el desarrollo de tareas más exigentes.

La destreza es otro desafío al que se enfrenta la robótica en los distintos sectores económicos y en la cual se está trabajando en distintos centros de investigación. Para el sector de la construcción en particular, labores como tender cableado exigen de una destreza mucho mayor a la que actualmente poseen los robots.

Otras desventajas se presentan en el sitio de trabajo donde se lleva a cabo la construcción. Una de ellas tiene que ver con las superficies irregulares; si bien la industria o los mismos laboratorios ofrecen superficies regulares para que los robots desarrollen sus labores, el 
sector de la construcción no puede hacer lo mismo. Esto hace que el robot pierda el sistema de referencia en su extremo y no pueda ejercer adecuadamente su labor, reduciendo precisión en la misma. Las inclemencias climáticas se pueden volver también un problema para esta tecnología, ya que se debe tener en cuenta que las labores en ocasiones han de ser en entornos abiertos, lo cual hace que la operación del robot se pueda ver comprometida.

\section{GEOMETRÍAS COMPLEJAS}

La robótica permite que los arquitectos tengan más herramientas para que lleven sus diseños a la realidad; generando geometrías complejas que hace unos años no era posible desarrollarlas, ya fuera por la falta de precisión del humano o por el excesivo costo que generaba desarrollarlas.

En la Escuela Politécnica Federal de Zurich (ETHZ), investigadores elaboraron una pared de madera con pequeñas piezas de distintos grosores en el año 2012 y una pared en ladrillo con forma de onda en 2016. Para ello se emplearon dos plataformas móviles: dimRob (Helm, Ercan, Gramazio y Kohler, 2012) e IF (Dörfler et al., 2016). Si bien el concepto de estos dos sistemas es el mismo, IF trae algunas mejoras sobre su predecesora dimRob; su precisión aumenta y la intervención humana se reduce considerablemente ya que no tiene que ser reposicionada manualmente.

Estas dos plataformas se encuentran equipadas con brazos robóticos, sensores (escáneres 2D, 3D e IMU), sistemas de control, software (Grasshopper Rhinoceros, ROS, ABB Robot Control y Python) y pinzas de vacío. El sistema permite conocer su propia localización dentro del entorno de fabricación y ensamblar componentes de construcción de una manera precisa en el espacio (Sandy et al., 2016).
Mallas de polímero para procesos de moldeo en estructuras de concreto, pueden ser desarrolladas a través de un método de tejido robótico (Hack, Lauer, Gramazio y Kohler, 2014). El sistema está provisto de un brazo robótico de 6 grados de libertad (GDL), una boquilla para extrudir el material y un subsistema de enfriamiento; este permite la construcción de geometrías complejas y la optimización de recursos.

Haciendo uso de la misma plataforma mencionada previamente (IF); se diseñó, desarroIló y evaluó una herramienta final (end-effector) para el brazo robótico de esta; la cual permite fabricar prototipos de mallas en alambre de distintos diámetros. Esto se elaboró con el fin de construir estructuras de concreto en acero reforzado que respondieran a geometrías complejas que son de difícil construcción con las actuales técnicas de fabricación (Kumar et al., 2017).

Es posible lograr curvaturas complejas a través de una producción manual o de un fresado en $\mathrm{CNC}$ a gran escala, pero ninguno de estos dos métodos es económico. Ahora bien, robotic hotwire cutting (RHWC) rompe con esta tendencia (McGee, Feringa y Sondergaard, 2013). Teniendo en cuenta el método RHWC, Sondergaard propone la utilización de una cuchilla en caliente (hotblade) para realizar cortes en distintos tipos de materiales.

Esta es montada entre dos brazos robóticos, lo cual ayuda a definir su posición y orientación; de esta forma, es posible determinar la geometría del objeto. Un robot más soporta la pieza que se desea cortar y la mueve linealmente en dirección de la cuchilla. El corte de la superficie para generar las curvaturas se ha de realizar por segmentos, esto teniendo en cuenta las dimensiones del objeto a cortar, así como la inestabilidad en la cuchilla (Sondergaard et al., 2016). 


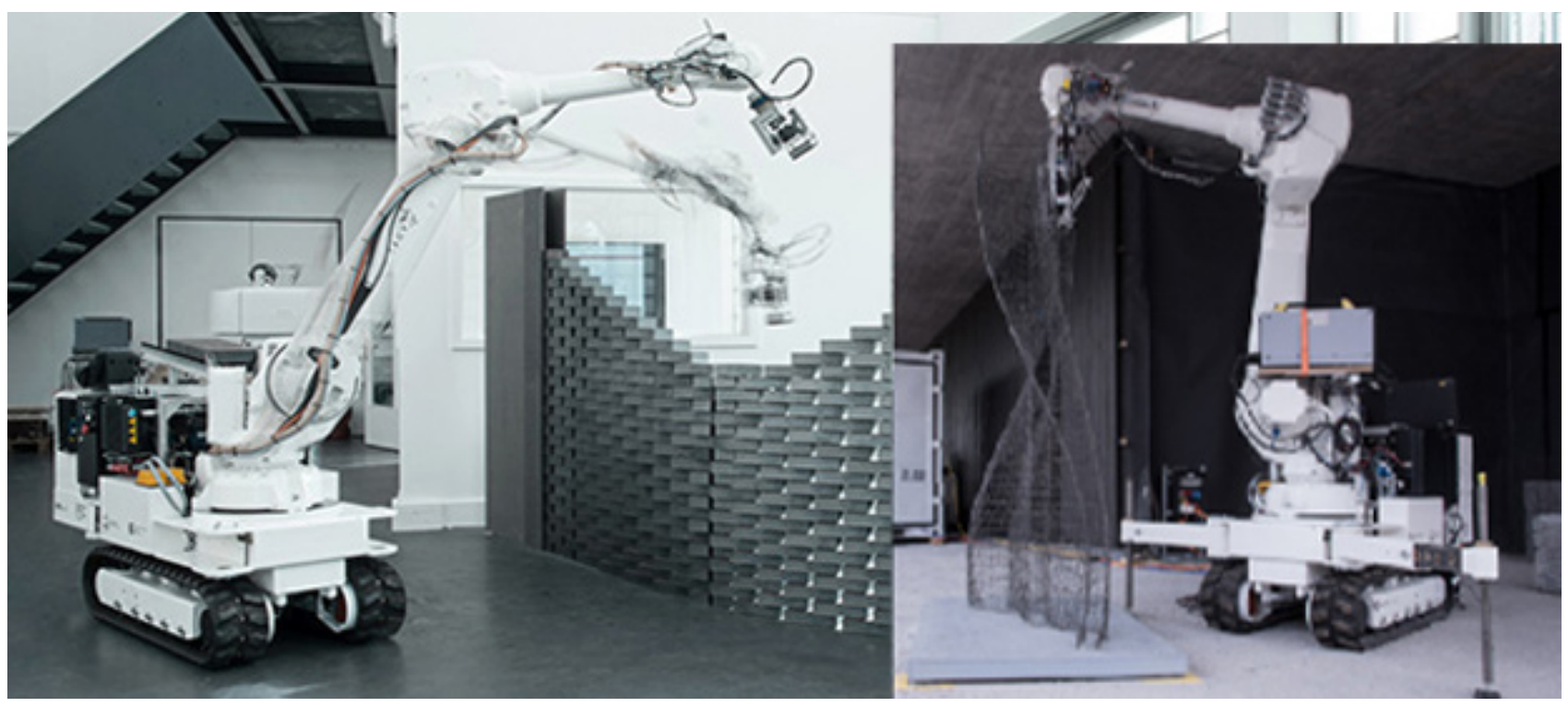

Figura 1. Plataforma robótica IF desarrollada por ETHZ (izq) End-effector para elaboración de prototipos de mallas de alambre (der). Fuente: Ajustado de (Dörfler et al., 2016) y (Kumar et al., 2017)

Las impresoras 3D tienen limitantes con respecto al volumen que pueden construir, lo cual hace que los diseños sean pensados en pequeñas piezas o módulos. En el sector de la construcción se complica aún más el uso de estas impresoras debido a las dimensiones en las cuales se trabajan; sin embargo, el uso de brazos robóticos o plataformas móviles facilita la creación de sistemas de impresión 3D que construyan estructuras en una sola pieza.

Además, si se combinan robos industriales con técnicas basadas en extrusión, como contour crafting (Khoshnevis, Hwang, Yao y Yeh, 2006), se obtienen estructuras en concreto sin la necesidad de utilizar encofrados (NematoIlahi, Xia y Sanjayan, 2017). El MIT propone utilizar un sistema robusto que permita fabricar en el sitio de trabajo, estructuras arquitectónicas personalizadas utilizando información del entorno. El sistema se compone por una plataforma móvil y un brazo robótico que a su vez está compuesto por un brazo eléctrico y uno hidráulico. El experimento que se llevó a cabo para evaluar su operación fue construir un domo de 14,6 metros y 3,7 metros de alto; con un tiempo de 13,5 horas (Keating, Leland, Cai y Oxman, 2017).

Bhooshan, Ladinig, Mele y Block (2019) proponen utilizar un brazo robótico que realice un proceso de impresión 3D por capas a gran escala utilizando concreto, generando así una estructura esquelética en este material. A diferencia de imprimir capa por capa de forma vertical (método convencional) desde un robot industrial, Zhang et al. (2015) sugieren un generador de ruta para superficies curvas, imprimiendo en ángulo estas mismas.

Por otro lado, tener distintos robots trabajando sobre una misma estructura permite acelerar el tiempo de producción, así como construir estructuras más fuertes y complejas. Inclusive, se puede pensar en utilizar enjambres robóticos de este tipo para construir en áreas remotas o de difícil acceso (Zhang et al., 2018). Fabricar estructuras de concreto de grandes dimensiones en el área de construcción se vuelve una ventaja, ya que evita el problema de movilizar pesos considerables (Lim et al., 2012). 
Al hablar de las geometrías complejas para paneles arquitectónicos, regularmente se piensa en la utilización de moldes; sin embargo, como se mencionó previamente, un robot industrial puede recrear geometrías complejas con gran precisión y en menor tiempo; empleando distintas herramientas y trayectorias.

Bard, Cupkova, Washburn y Zeglin (2018) utilizan este principio en paneles de concreto: primero, deposita el material a través de una boquilla ubicada en el extremo del robot, y segundo, remueve, a través de un barrido, pequeñas cantidades de material en la superficie de la pieza. Otra técnica es utilizar lycra como encofrado para verter concreto dentro de ella, apoyándose en brazos robóticos que trabajan cooperativamente para que la estiren y la posicionen según las necesidades de diseño.

Las piezas fabricadas son de tres extremidades; dos de ellas están sujetas a cada uno de los robots y la restante se encuentra sujetada a la armadura, la cual también se utiliza para realizar el ingreso del concreto. Este tipo de piezas permiten construir fachadas, paredes e inclusive esculturas (Culver, Koerner y Sarafian, 2016).

Dejando a un lado el uso de brazos robóticos, Shaffer (2017) presenta un encofrado robotizado, semejante a una cadena, el cual crea una superficie de fundición que puede moverse y cambiar de forma.

El mecanismo está compuesto por distintos eslabones enlazados entre sí, los cuales son accionados por motores en cada uno de sus ejes; igualmente el accionamiento de cada uno de estos motores se puede realizar remotamente a través de una app. Esta propuesta puede mejorar la respuesta a requerimientos arquitectónicos a través de personalizado y variabilidad en cada desarrollo.

\section{SISTEMAS RETROALIMENTADOS Y ALGORITMOS DE PLANIFICACIÓN}

Como se pudo entender de la anterior sección, se abarcaron diversos sistemas en lazo abierto que permiten construir estructuras con geometrías complejas, y esto gracias a que los procesos de fabricación tienden a ser predeterminados. Sumado a lo anterior, en los últimos años se ha evolucionado hacia sistemas en lazo cerrado para robots en el sector de la construcción; por tanto, sensores externos se han vueltos aliados primordiales en este nuevo desafío.

Lo que se busca con esto es que los robots sean capaces de recibir información del entorno de trabajo (área de construcción), la procesen y actúen en función de esta. Dubor et al. (2016) plantean un marco de trabajo para la fabricación robótica el cual logre: enlazar datos, conocer el comportamiento del material, interactuar, definir protocolos y tener retroalimentación; además, combinar herramientas paramétricas (Rhinoceros 3D + Grasshopper 3D) con plugins CAM (Kuka \prc) para explorar el potencial y las limitaciones que tiene el proceso de fabricación robótica.Los sensores ayudan a conocer las variables del entorno que pueden afectar la fabricación de la pieza o estructura, como temperatura, velocidad de extrusión del material, humedad, presión, entre otras; así como optimizar la trayectoria del brazo robótico y trabajar de forma colaborativa con humanos o inclusive con otros robots.

Por otro lado, los sensores dan a conocer los avances de la obra y el comportamiento dinámico del material de construcción; Sutjipto et al. (2019) consideran que mediante una cámara en el extremo de un brazo robótico se determina si una locación del espacio de trabajo está ocupada por material previamente depositado. Igualmente, el uso de cámaras, técnicas de visión y brazos robóticos posibilita identificar y manipular ob- 


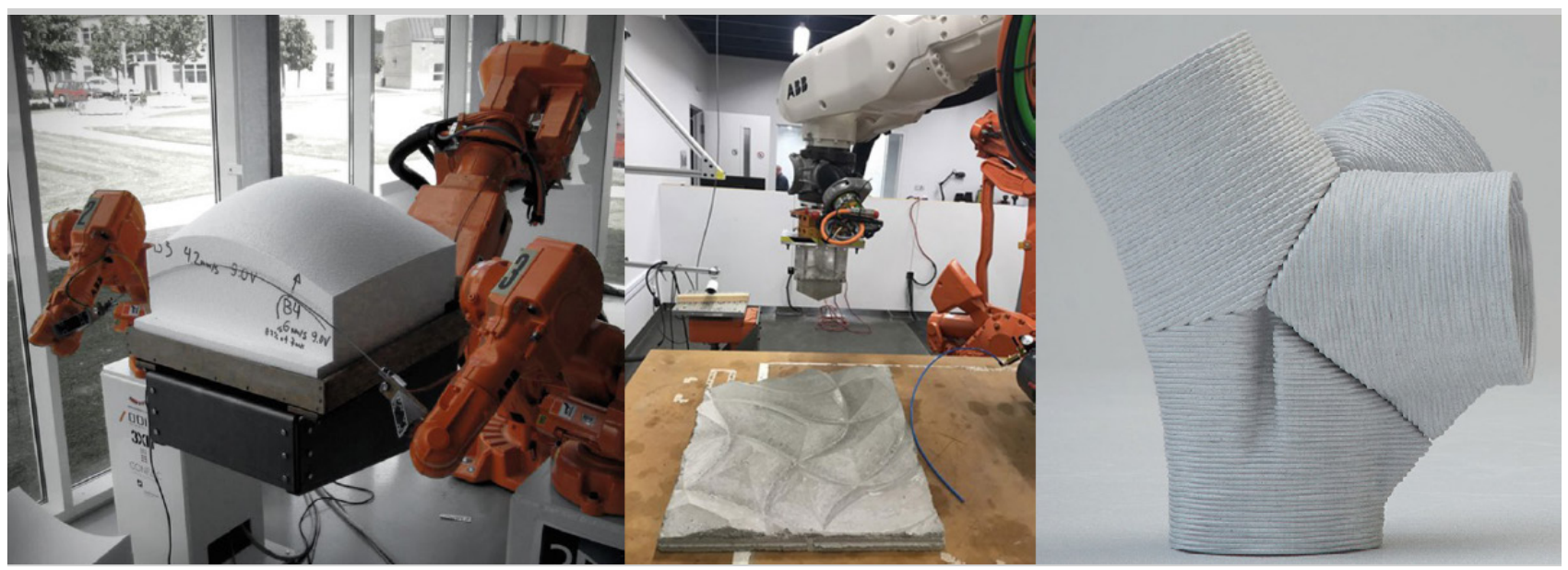

Figura 2. Hotblade para la fabricación de superficies curvas (izq.). Impresión 3D en concreto (med.). Módulos en concreto fabricados con un brazo robótico (der.)

Fuente: Ajustado de (Sondergaard et al., 2016), (Bard, Cupkova, Washburn y Zeglin, 2018) y (Bhooshan, Ladinig, Mele y Block, 2019)

jetos (Bueno, Arteaga y Candea, 2013), así como ensamblar cada uno de estos en una estructura prediseñada (Feng et al., 2014; Feng et al., 2015).

La robótica cooperativa también ha hecho sus aportes en el sector de la construcción; un ejemplo de ello es Termes (Petersen, Nagpal y Werfel, 2011), un sistema autónomo multirrobot bioinspirado que permite construir estructuras 3D de acuerdo con las especificaciones del usuario; además tiene la capacidad de responder ante cambios en la obra. El sistema se compone de robots móviles que se comunican entre ellos y de bloques especializados para su fácil manipulación, así como de un algoritmo de control descentralizado. Otro ejemplo es Fiberbots (Kayser et al., 2018), el cual es un sistema bioinspirado de robots idénticos que construyen distintas partes de la misma estructura en paralelo. Estos crean tubos compuestos que se pueden escalar y extender, controlando la curvatura estos. El diseño del robot se divide en tres tareas: manipulación del material compuesto, movilidad y coordinación entre robots.
De acuerdo con el trabajo cooperativo que puede hacerse entre robots, la planificación de rutas (path planning) es otra técnica que ha tomado relevancia no solo en la robótica móvil (Arbulú, Martinez y Montiel, 2015), (Martinez, Orjuela y Arbulú, 2016), sino también en brazos robóticos empleados en distintos sectores, entre ellos la construcción. Esta permite realizar tareas, como ensamblar piezas o evitar obstáculos, ya sean estos, la misma estructura que se está fabricando u otro robot que esté trabajando en paralelo.

Existen algoritmos que resuelven eficientemente la secuencia de extrusión, las poses del efector final del robot, la posición de las articulaciones y las trayectorias transitorias para cerchas espaciales (Huang, Garret y Mueller, 2018). Un ejemplo más puntual es que mientras un robot sostiene la estructura temporalmente, el otro posiciona una pieza dentro de esta (Gandia et al., 2019).

Existen sistemas robustos donde interactúan robots de $6 \mathrm{GDL}$, los cuales a su vez son integrados a una base con movimiento en tres ejes que ensamblan estructuras de ma- 


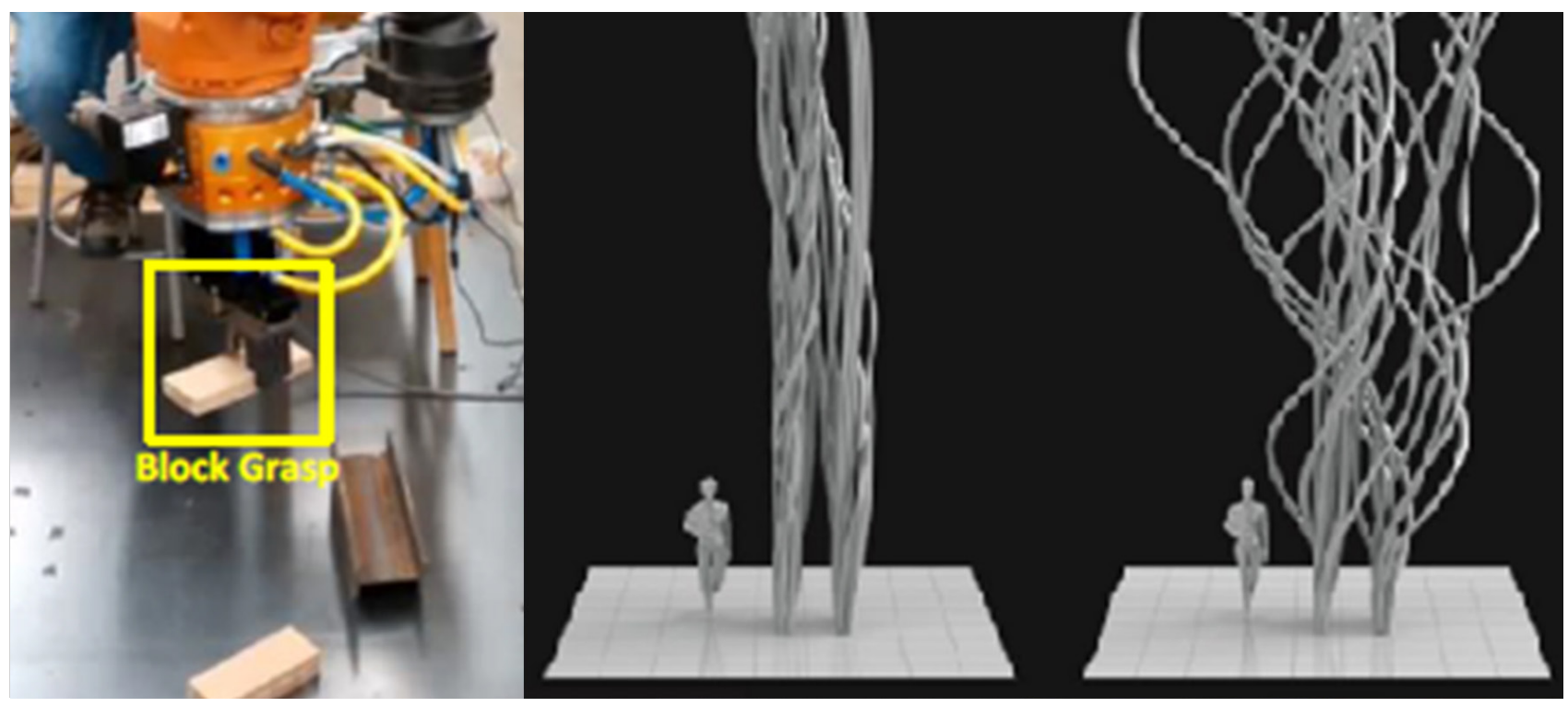

Figura 3. Sistema de visión y brazo robótico (izq.). Fiberbots (der.)

Fuente: Ajustado de (Feng et al., 2014) y (Kayser et al., 2018.)

dera (Thoma et al., 2018) o estructuras de metal (Parascho et al., 2017); haciendo uso de diversas herramientas (fresado, taladrado, cortado, soldadura, etc.); y soportados en algoritmos de planificación de rutas al ser un trabajo cooperativo.

Trabajar junto a robots fue la idea que tuvo Shimizu Corporation, además de reducir el agotamiento y las tareas repetitivas, así como el de aumentar la productividad y el entusiasmo de las generaciones más jóvenes al trabajar en el sector de la construcción. La compañía actualmente cuenta con tres robots para este sector: el primero se especializa en soldar autónomamente sin la intervención humana; el segundo se encarga de instalar techos, detectando su locación a través de imágenes y LIDAR (laser imaging detection and ranging); y el último es un robot móvil que transporta material, planifica rutas para evitar obstáculos y entra a ascensores para moverse entre pisos (Shimizu Corporation, 2019). Empleando igualmente la inteligencia artificial, además de la realidad virtual, la startup SE4 utiliza estas herra- mientas para enseñarle a brazos robóticos a excavar o apilar bloques para la construcción de estructuras en el espacio. El software es entrenado en un entorno simulado, lo que permite posteriormente al robot desarrollar tareas en un entorno real. El problema radica en los retrasos en la comunicación, así como de no tener agentes inteligentes en el espacio de trabajo (SE4 Inc., 2019).

\section{TRABAJO PESADO}

Si bien hasta el momento se han descrito en su gran mayoría brazos robóticos industriales orientados al sector de la construcción -adaptándoles boquillas de extrusión, sensores, algoritmos de planificación de rutas, entre otros elementos-, también se presentan otras configuraciones robóticas que ayudan en tareas más robustas. En la actualidad, diversos sistemas robóticos son operados fuera del área de construcción, debido a lo explicado previamente con respecto a la complejidad que traen estos entornos de trabajo; además el ensamblaje en este sector aún se realiza en el área de trabajo; sin 
embargo, se han dado adelantos en traer la fabricación al sitio de trabajo, con el principal objetivo de reducir costos en la obra.

El sistema autónomo SPIDERobot (Moreira et al., 2014) se desarrolló para que trabajara dentro del espacio de la construcción. El hardware de este robot lo componen cuatro cables ubicados en las esquinas superiores del marco de trabajo, los cuales se recogen y se extienden a través de motores; una plataforma móvil a donde convergen cada uno de los cables, una pinza y un sistema de visión (Sousa et al., 2016). El robot posee $4 \mathrm{GDL}$, los cuales le permiten trasladarse en coordenadas $(x, y, z)$; así como una rotación en el eje $z$, la cual está asociada a la pinza. Configuraciones semejantes a SPIDERobot son las realizadas por investigadores de la Universidad de Laval (Barnett y Gosselin, 2015); e investigadores de IAAC y Tecnalia (Izard et al., 2017). La primera de estas se centra en la construcción de una estatua de 2,16 m con una precisión de $1 \mathrm{~cm}$, utilizando espuma de poliuretano como material objeto. La segunda se centra en el desarrollo del robot paralelo Cogiro; entre sus características se resalta que tiene un espacio de trabajo mucho más amplio que el de un robot industrial convencional y su posicio-

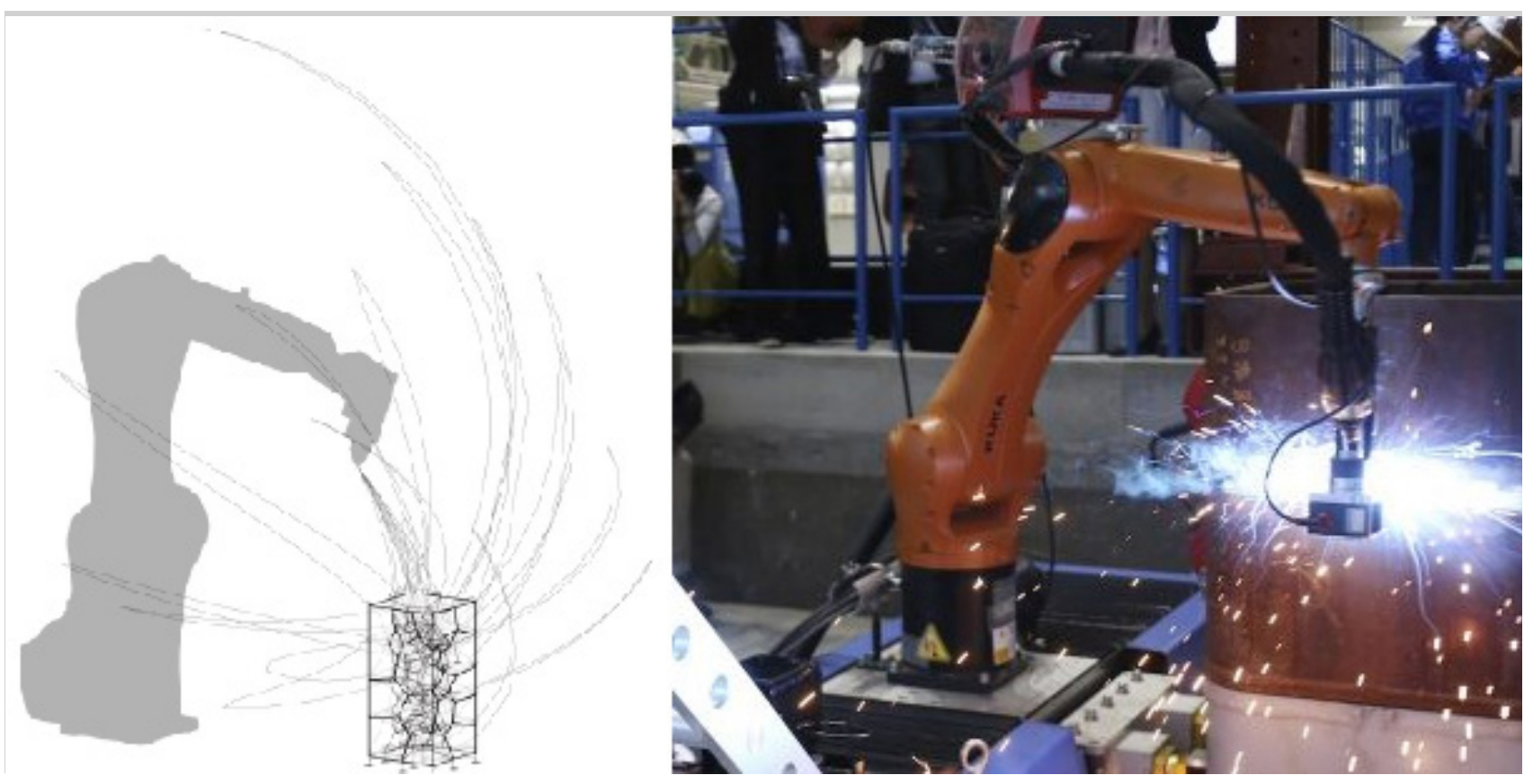

Figura 4. Planificación de rutas (izq.). Robot soldador autónomo de SHIMIZU (der.) Fuente: Ajustado de (Huang, Garret y Mueller, 2018) y (Shimizu Corporation, 2019)

namiento sigue siendo determinado por el enrollamiento de cables al igual de SPIDERobot. En esta investigación se crearon paredes de arcilla con una geometría ondulante, a través de capas superpuestas unas sobre otras; validando trayectorias rectas y curvas, apilamiento de capas, velocidad de impresión y tiempo de secado del material.
Uno de los inconvenientes que tienen los robots industriales es que son pesados y voluminosos; lo cual los hace de difícil despliegue y desplazamiento en algunas áreas de construcción. La plataforma robótica presentada por Dharmawan et al. (2017) permiten que un robot industrial de bajo peso (Universal Robot UR10) pue- 
da navegar entre pisos (sitio de trabajo), otorgándole al brazo robótico 1 GDL extra. El sistema se integra fácilmente a andamios, realiza labores de soldadura e ingresa a espacios estrechos exclusivos para el ser humano. Otro robot colaborativo es SAM100, el cual trabaja de la mano con el albañil en el posicionamiento de ladrillos en construcciones. Entre sus características se encuentran disminuir el costo de la instalación, incrementar la productividad (posiciona 300 ladrillos/hora), reducir el esfuerzo de levantamiento (hasta un $80 \%$ ) y mejora la precisión. El sistema lo compone un brazo robótico, inyector de concreto, sistema de alimentación de ladrillos, sensores y software propio de la empresa; además funciona con gas propano. Requiere de dos técnicos: uno que supervisa su correcto desempeño y otro que se encarga de suministrar los ladrillos al sistema (Construction Robotics, 2019).

Otra labor que ocupa gran atención en el sector de la construcción es la demolición. Esta es una de las tareas más riesgosas en este sector, razón por la cual los robots que desempeñan esta tarea ocupan actualmente el $90 \%$ (RIA, 2019) del mercado total de robots utilizados en el sector de la cons- trucción. El automatizar esta tarea permite mantener alejados a los trabajadores de situaciones riesgosas, haciendo que estos se concentren en tareas más productivas.

Una de las grandes compañías que se dedican al desarrollo de robots para actividades de demolición es Husqvarna; sus robots son teleoperados, tienen un sistema de locomoción para desplazarse a lo largo de la obra y, además, están provistos con un brazo que permite intercambiar herramientas dependiendo de la tarea que se quiera realizar (excavar, palear, agarrar, zanjar, aplastar, remover, etc.). Su alcance llega hasta los 5,5 metros, tienen una alta relación potencia/peso, pueden pasar por puertas de tamaño estándar y tienen un bajo centro de masa que les permite mayor estabilidad (Husqvarna, 2019).

Otro robot con características muy semejantes es el de la compañía Brokk; los niveles de ruido y vibraciones que esta máquina produce son bajos, lo cual lo hace ideal para trabajar en horarios de oficina o inclusive en áreas donde existan restricciones de ruido; además, a diferencia de otros sistemas, labores de cortado y perforado no requieren el uso de agua (Brokk, 2019). Los vehículos au-
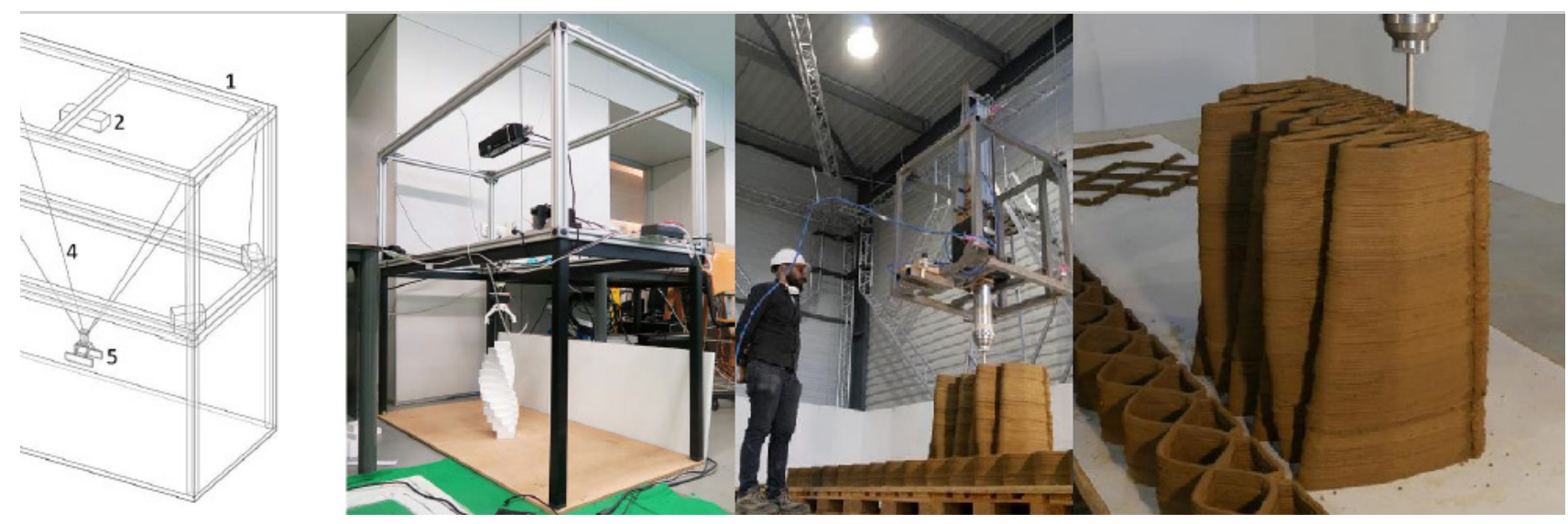

Figura 5. The SPIDERobot (izq.). Cogiro (der.)

Fuente: Ajustado de (Moreira et al., 2014) y (Izard et al., 2017) 
tónomos han tomado gran relevancia en los últimos años, atrayendo la atención de centros de investigación y empresas alrededor del mundo; sin embargo, aún se encuentran en desarrollo debido al entorno complejo y altamente dinámico al cual se enfrentan. Partiendo de ese mismo concepto, pero en un entorno más controlado, Built Robotics ha venido desarrollando bulldozers, excavadoras y minicargadores autónomos, los cuales están en la capacidad de realizar las mismas tareas (excavar, empujar, levantar, cargar, manipular, zanjar, etc.) que desarroIlan los vehículos convencionales. Cada uno de estos robots está provisto de sensores que permiten detectar personas, animales y otros vehículos; tienen un sistema donde el robot está confinado a trabajar en un lugar delimitado, y el supervisor puede parar la operación en cualquier momento que desee (Built Robotics Inc., 2019).

No solo la demolición tiende a ser una tarea riesgosa; entrar en contacto con un material como el asbesto también lo es. El proyecto Bots2Rec de Europa (Detert et al., 2017) y el trabajo desarrollado por Takenaka Corporation en Japón (Arai y Hoshino, 2010) buscan utilizar robots móviles para remover autónomamente asbesto de superficies regulares, como paredes, techos, vigas y pisos. Entre las características físicas de los sistemas presentados se encuentran: herramientas abrasivas para la remoción, sensores de fuerza, plataformas móviles, brazos robóticos de 6 o $7 \mathrm{GDL}$, unidades de aspiración y finalmente, interfaces para ser teleoperados y supervisados. Estos sistemas evitan que operarios entren en contacto directo con este material contaminante, lo cual previene problemas de salud e incrementan el tiempo de remoción sobre las superficies, comparado con el de un humano.

Para el sector de la construcción también se han desarrollado exoesqueletos que permi-
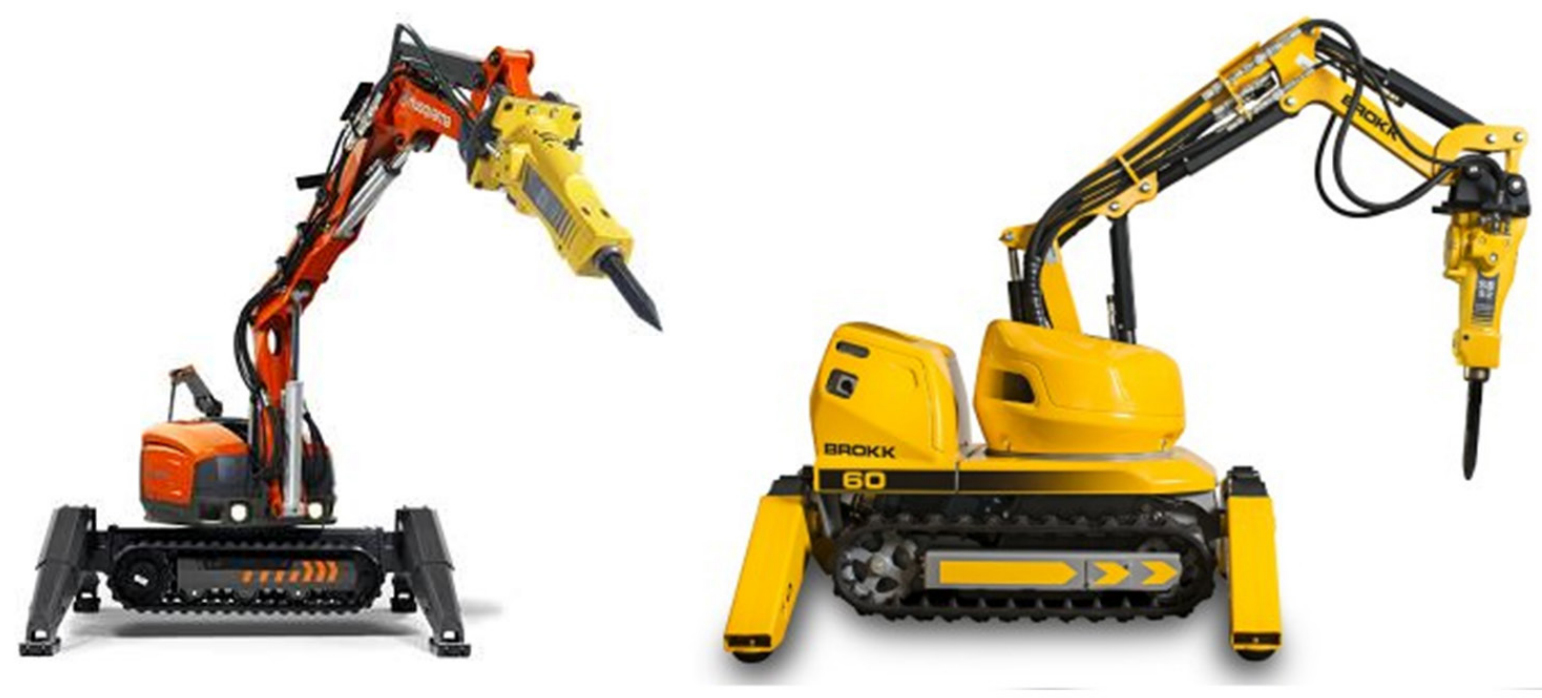

Figura 6. Robot de demolición de Husqvarna (izq.). Robot de demolición de Brokk (der.) Fuente: Ajustado de (Husqvarna, 2019) y (Brokk, 2019) 
ten maximizar características físicas en el ser humano, reduciendo la accidentabilidad y posibles lesiones que se puedan presentar a lo largo de los años por realizar tareas repetitivas y manipulación de altos pesos en el lugar de trabajo. La compañía Cyberdyne desarrolló el sistema HAL, un exoesqueleto que ayuda a incrementar la fuerza del usuario y reducir el estrés aplicado en la espalda por el levantamiento de elementos pesados como los que se pueden encontrar en una obra (Cyberdyne Inc., 2019). Fortis es otro exoesqueleto que permite aliviar la carga del usuario y reducir su fatiga muscular a través de biomecánica; gracias a ello, aumenta la productividad en la obra, y mejora fuerza y resistencia del usuario; adicionalmente, es un sistema que no necesita de una fuente de alimentación para ser utilizado (Lockheed Martin Corporation, 2019).

Los robots humanoides han empezado a ser objeto de investigación para el sector de la construcción, con el fin de que puedan replicar el movimiento humano en entornos de construcción complejos. Se ha avanzado en distintos frentes con el uso de brazos robóticos y robots móviles; algunos ejemplos han sido mencionados en párrafos anteriores, sin embargo todavía es necesaria la intervención humana para unificar las construcciones. HRP-5P es un robot albañil diseñado y desarrollado por Japan's National Institute of Advanced Industrial Science and Technology, capaz de generar mapas 3D del entorno, detectar objetos, manipular hojas de drywall de hasta $13 \mathrm{~kg}$, así como de emplear herramientas para ensamblarlas (National Institute of Advanced Industrial Science and Technology [AIST], 2018).

\section{CONCLUSIONES}

La robótica continuará expandiéndose en los diversos sectores económicos y definiti- vamente el sector de la construcción es uno de ellos. Un nuevo uso de los brazos robóticos industriales ha sido descubierto; emplearlos como herramientas que fabrican piezas con geometrías complejas y con un alto grado de precisión es posible, dejando al humano concentrarse en la creatividad del diseño. Si bien, inicialmente, el brazo robótico tenía limitantes en construir espacios amplios o piezas de volúmenes considerables, en la actualidad se tienen plataformas robóticas móviles que permiten ampliar su espacio de trabajo.

El desarrollo de proyectos de construcción se ha vuelto una tarea multidisciplinar, donde no solo se encuentran implicados arquitectos, ingenieros civiles y albañiles, sino también personas con conocimientos en robótica, programación, CAD/CAM, inteligencia artificial, entre otros.

La sociedad en la actualidad se está moviendo hacia el desarrollo de habilidades cognitivas, dejando las tareas repetitivas, de alta precisión o que implican fuerza, a sistemas robóticos que las pueden desempeñar mucho mejor que un ser humano. Debido a esto se está presentando una reducción significativa y un envejecimiento en la población encargada de labores físicas.La robótica no solamente puede ser aprovechada para la fabricación de arquitectura interesante, sino también de mejorar las condiciones del trabajador (ergonomía, seguridad y satisfacción); además de realizas construcciones más simples, más eficientes y menos costosas (tanto en tiempo como en material de obra).

Debido al hecho de que el sector de la construcción es un entorno no estructurado y dinámico; el empleo de sensores en plataformas robóticas permite identificar cambios en el ambiente y así mismo responder ante cambios imprevistos. Otra gran herramienta que viene para estos sistemas es 


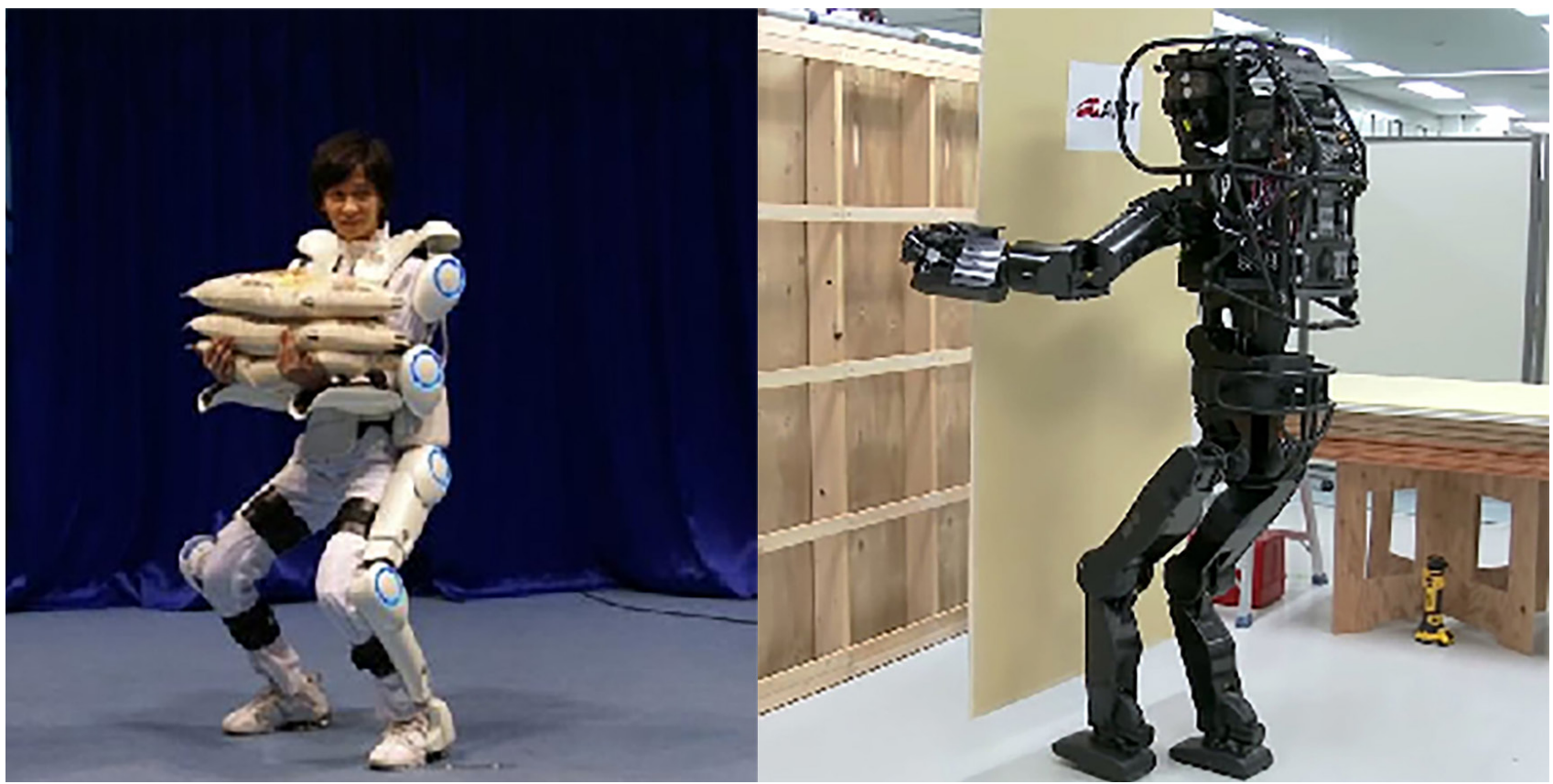

Figura 7. Exoesqueleto de Cyberdyne (izq.). Robot humanoide HRP-5P (der.)

Fuente: Ajustado de (Cyberdyne Inc., 2019) y (National Institute of Advanced Industrial Science and Technology [AIST], 2018)

el uso de la inteligencia artificial; la cual permitirá aprender tanto del humano como de otros robots y del propio entorno. Estos dos elementos harán de los robots, sistemas más inteligentes y autónomos, con la capacidad de responder ante un sector cambiante como es el de la construcción.

\section{FINANCIAMIENTO}

Corporación Unificada Nacional de Educación Superior (CUN).

\section{REFERENCIAS}

ABI Research (2019). Construction and mining represent the next frontier of robotics adoption thanks to automation technologies advances. Nueva York. Recuperado de https://www.abiresearch.com/press/ construction-and-mining-represent-ne$\mathrm{xt}$-frontier-robotics-adoption-thanks-automation-technology-advances/
Arai, M. y Hoshino, H. (2010). Robot system for removing asbestos sprayed on beams. Japón. Recuperado de http://www.irbnet. de/daten/iconda/CIB_DC23562.pdf

Arbulú Saavedra, M. R., Martínez Santa, F., \& Montiel Ariza, H. (2015). Metodología para el uso de la técnica de localización de raíces en la planeación de rutas para robots móviles. Revista Tecnura, 19(46), 49-64. DOI: https://doi.org/10.14483/ udistrital.jour.tecnura.2015.4.a04

Bard, J., Cupkova, D., Washburn, N. y Zeglin, G. (2018). Robotic concrete surface finishing: a moldless approach to creating thermally tuned surface geometry for architectural building components using Profile-3D-Printing. Construction Robotics, 2, 53-65.

Barnett, E. y Gosselin, C. (2015). Large-scale $3 \mathrm{D}$ printing with a cable-suspended robot. Additive Manufacturing, 7(1), 27. DOI: https://doi.org/10.1016/j.addma.2015.05.001 
Bhooshan, S., Ladinig, J., Mele, T. y Block, P. (2019). Function Representation for Robotic 3D Printed Concrete. Robotic Fabrication in Architecture, Art and Design 2018, 98-109.

Brokk (2019). Construction. EE. UU. Recuperado de https://www.brokk.com/us/industry/construction/

Bueno, M., Arteaga, M. y Candea, A. (2013). Modelado de sistemas de visión en 2D y 3D: Un enfoque hacia el control de robots manipuladores. Revista Tecnura, 17(37), 12-21. DOI: https://doi.org/10.14483/ udistrital.jour.tecnura.2013.3.a01

Built Robotics Inc. (2019). About. EE. UU. Recuperado de https://www.builtrobotics.com/

Construction Robotics (2019). SAM100. EE. UU. Recuperado de https://www.construction-robotics.com/sam100/

Culver, R., Koerner, J. y Sarafian, J. (2016). Fabric forms: The robotic positioning of fabric formwork. Robotic Fabrication in Architecture, Art and Design 2016, 106121.

Cyberdyne Inc. (2019). HAL. Japón. Recuperado de https://www.cyberdyne.jp/english/products/HAL/index.html

Detert, T., Eddine, S., Fauroux, J., Haschke, T., Becchi, F., Corves, B., Guzman, R., Herb, F., Linéatte, B., Martin, D. y Barrado, M. (2017). Bots2 ReC: Introducing mobile robotic units on construction sites for asbestos rehabilitation. Construction Robotics, 1, 29-37.

Dharmawan, A., Sedoro, B., Foong, S. y Soh, G. (2017). An agile robotic system mounted on scaffold structures for on-site construction work. Construction Robotics, 1, 15-27.
Dörfler, K., Sandy, T., Giftthaler, M., Gramazio, F., Kohler, M. y Buchli, J. (2016). Mobile robotic brickwork-automation of a discrete robotic fabrication process using an autonomous mobile robot. En D. Reinhardt, R. Saunders y J. Burry (Ed.), Robotic Fabrication in Architecture, Art and Design 2016 (pp. 204-217). Springer International Publishing.

Dubor, A., Camprodom, G., Bello, G., Reinhardt, D., Saunders, R., Dunn, K., Niemela, M., Horlyck, S., Alarcon, S., Wozniak, D. y Watt, R. (2016). Sensor and workflow evolutions: Developing a framework for instant robotic toolpath revision. En D. Reinhardt, R. Saunders y J. Burry (Ed.), Robotic Fabrication in Architecture, Art and Design 2016 (pp. 410-425). Springer International Publishing.

Feng, C., Xiao, Y., Willete, A., McGee, W. y Kamat, V. (2015). Vision guided autonomous robotic assembly and as-built scanning on structured construction sites. Automation in Construction, 59, 128-138. DOI: https://doi.org/10.1016/j. autcon.2015.06.002

Feng, C., Xiao, Y., Willete, A., McGee, W., y Kamat, V. (2014). Towards autonomous robotic in-situ assembly on structured construction sites using monocular vision. En Proceedings of the 31st International Association for Automation and Robotics in Construction (pp. 163-170). Sídney, Australia.

Gandia, A., Parascho, S., Rust, R., Casas, G., Gramazio, F. y Kohler, M. (2019). Towards automatic path planning for robotically assembled spatial structures. En J. Willmann, P. Block, M. Hutter, K. Byrne y T. Schork (Ed.), Robotic Fabrication in Architecture, Art and Design 2018 (pp. 59-73). Springer International Publishing. 
Hack, N., Lauer, W., Gramazio, F. y Kohler, M. (2014). Mesh-Mould: Robotically fabricated spatial meshes as reinforced concrete formwork. Architectural Design. 84(3)

Helm, V., Ercan, S., Gramazio, F. y Kohler, M. (2012). Mobile robotic fabrication on construction sites: dimRob. En Proceedings of the 2012 IEEE/RSJ International Conference on Intelligent Robots and Systems (pp. 4335-4341). Vilamoura, Algarve, Portugal.

Huang, Y., Garret, C. y Mueller, C. (2018). Automated sequence and motion planning for robotic spatial extrusion of 3D trusses. Construction Robotics, 2, 15-39.

Husqvarna (2019). Demolition Robots. Suecia. Recuperado de https://www.husqvarnacp.com/global/machines/demolition-robots/

Izard, J., Dubor, A., Hervé, P., Cabay, E., Culla, D., Rodriguez, M. y Barrado, M. (2017). Large-scale 3D printing with cable-driven parallel robots. Construction Robotics, 1, 69-76.

Kayser, M., Cai, L., Falcone, S., Bader, C., Inglessis, N., Darweesh, B. y Oxman, N. (2018). Fiberbots: An autonomous swarm-based robotic system for digital fabrication of fiber-based composites. Construction Robotics, 2, 67-79.

Keating, S., Leland, J., Cai, L. y Oxman, N. (2017). Toward site-specific and self-sufficient robotic fabrication on architectural scales. Science Robot, 2, 1-15.

Khoshnevis, B., Hwang, D., Yao, K. y Yeh, Z. (2006). Mega-scale fabrication by contour crafting. International Journal Industrial and Systems Engineering, 1(3), 301320.
Kumar, N., Norman, H., Doerfler, K., Walzer, A., Rey, G., Gramazio, F., Kohler, M. y Buchli, J. (2017). Design, development and experimental assessment of a robotic end-effector for non-standard concrete applications. En 2017 IEEE International Conference on Robotics and Automation (ICRA) (pp. 1707-1713). Singapore, Singapore. DOI: https://doi.org/10.1109/ ICRA.2017.7989201

Lim, S., Buswell, R., Le, T., Austin, S., Gibb, A. y Thorpe, T. (2012). Developments in construction-scale additive manufacturing processes. Automation in Construction, 21, 262-268.

Lockheed Martin Corporation (2019). Exoskeleton. EE. UU. Recuperado de https://www. lockheedmartin.com/en-us/products/exoskeleton-technologies/military.html

Martínez S., F.; Orjuela R., S. y Arbulú S., M. (2017). Global navigation approach for assistant robot. Revista Tecnura, 21(51), 105-117. DOI: https://doi.org/10.14483/ udistrital.jour.tecnura.2017.1.a08

McGee, W., Feringa, J. y Sondergaard, A. (2013). Processes for an architecture of volume. En S. Brell-Cockan y J. Braumann (Ed.), Robotic Fabrication in Architecture, Art and Design 2012 (pp. 62-71). Springer International Publishing.

Moreira, E., Pinto, A.M., Costa, P., Moreira, A.P., Veiga, G., Lima, J., Sousa, J.P. y Costa, P. (2014). Cable robot for non-standard architecture and construction: A dynamic positioning system. En IEEE International Conference on Industrial Technology (ICIT) (pp. 3184-3189). Sevilla, España. DOI: https://doi.org/10.1109/ ICIT.2015.7125568. 
National Institute of Advanced Industrial Science and Technology (2018). Development of a humanoid robot protoype, HRP-5P, Capable of Heavy Labour. Japón. Recuperado de https:// www.aist.go.jp/aist_e/list/latest_research/2018/20181116/en20181116. html

Nematollahi, B., Xia, M. y Sanjayan, J. (2017). Current progress of 3D concrete printing technologies. En Proceedings of the 34th Symposium on Automation and Robotics in Construction (ISARC) (pp. 260-267). Taipéi, Taiwán. DOI: https:// doi.org/10.22260/ISARC2017/0035

Parascho, S., Gandia, A., Mirjan, A., Gramazio, F. y Kohler, M. (2017). Cooperative fabrication of spatial metal structures. En A. Menges, B. Sheil, R. Glynn y M. Skavara (Ed.), Fabricate 2017 (pp. 24-29). UCL Press. DOI: https://doi.org/10.3929/ ethz-b-000219566

Petersen, K., Nagpal, R. y Werfel, J. (2011). Termes: An autonomous robotic system for three-dimensional collective construction. En Robotics: Science and Systems VII. 27 a 30 de junio, Los Ángeles, EE. UU.

Robotic Industries Association (RIA) (2019). Demolition Robots. Míchigan. Recuperado de https://www.robotics.org/service-robots/demolition-robots

Sandy, T., Giftthaler, M., Dorfler, K., Kohler, M. y Buchli, J. (2016). Autonomous repositioning and localization of an in situ fabricator. En Proceedings of the 2016 IEEE International Conference on Robotics and Automation (ICRA) (pp. 28522858). Estocolmo, Suecia. DOI: https:// doi.org/10.1109/ICRA.2016.7487449.
SE4 Inc. (2019). Space. Japón. Recuperado de http://se4.space

Shaffer, M. (2017). Developing robotic formwork: enhancing formwork mobility and variability through mechanization. Construction Robotics, 1, 77-83.

Shimizu Corporation. (2019). Construction. Japón. Recuperado de https://www. shimz.co.jp/en/topics/construction/ item12/

Sondergaard, A., Feringa, J., Norbjerg, T., Steenstrup, K., Brander, D., Graversen, J., Markvorsen, S., Baerentzen, A., Petkov, K., Hattel, J., Clausen, K., Jensen, K., Knudsen, L. y Kortbek, J. (2016). Robotic hot-blade cutting: An industrial approach to cost-effective production of double curved concrete structures. En D. Reinhardt, R. Saunders y J. Burry (Ed.), Robotic Fabrication in Architecture, Art and Design 2016 (pp. 150-164). Springer International Publishing.

Sousa, J., Palop, C., Moreira, E., Pinto, A., Lima, J., Costa, P., Costa, P., Veiga, G. y Moreira, P. (2016). The SPIDERobot: A cable-robot system for on-site in robotics. En D. Reinhardt, R. Saunders y J. Burry (Ed.), Robotic Fabrication in Architecture, Art and Design 2016 (pp. 230-239). Springer International Publishing.

Sutjipto, S., Tish, D., Paul, G., Vidal, T. y Schork, T. (2019). Towards feedback loops for robot-controlled additive manufacturing. En J. Willmann, P. Block, M. Hutter, K. Byrne y T. Schork (Ed.), Robotic Fabrication in Architecture, Art and Design 2018 (pp. 85-97). Springer International Publishing. 
Thoma, A., Adel, A., Helmreich, M., Wehrle, T., Gramazio, F. y Kohler, M. (2018). Robotic fabrication of bespoke timber frame modules. En J. Willmann, P. Block, M. Hutter, K. Byrne y T. Schork (Ed.), Robotic Fabrication in Architecture, Art and Design 2018 (pp. 447-458). Springer International Publishing.

Zhang, G., Mondesir, W., Martinez, C., Li, X., Fuhlbrigge, T. y Bheda, H. (2015). Robotic Additive Manufacturing along Curved Surface - a Step towards Freeform Fabrication. En Proceedings of the 2015 IEEE Conference on Robotics and Biomimetics (ROBIO) (pp. 721726). Zhuhai, China. DOI: https://doi. org/10.1109/ROBIO.2015.7418854.

Zhang, X., Li, M., Lim, J., Weng, Y., Tay, Y., Pham, H. y Pham, Q. (2018). Large-scale $3 \mathrm{D}$ printing by a team of mobile robots. Automation in Construction, 95, 98-106. 